\title{
COEFFICIENT EXTREMAL PROBLEMS FOR SCHLICHT FUNCTIONS
}

\author{
BY \\ J. T. POOLE(1)
}

I. Introduction. This pap $\mathbf{r}$ deals with extremal problems for the coefficients in the power series expansions of regular and schlicht functions, starlike functions, and the powers of these functions.

Definition 1.1. Let $S$ denote the class of functions

$$
f(z)=z+a_{2} z^{2}+a_{3} z^{3}+\cdots
$$

which are regular and schlicht in the unit circle $U=\{z:|z|<1\}$.

Let $\Sigma$ denote the class of functions

$$
\tilde{f}(z)=z+\tilde{a}_{0}+\frac{\tilde{a}_{1}}{z}+\frac{\tilde{a}_{2}}{z^{2}}+\cdots
$$

which are regular and schlicht in the exterior of the unit circle $V=\{z:|z|>1\}$ except for the simple pole at infinity.

Let $S^{*}$ denote the subclass of $S$ of functions which are also starlike in $U$, i.e., a function $f \in S$ is in $S^{*}$ if and only if

$$
\operatorname{Re}\left\{\frac{z f^{\prime}(z)}{f(z)}\right\}>0
$$

for all $z \in U$.

Likewise, let $\Sigma^{*}$ denote the subclass of $\Sigma$ of functions which are also starlike in $V$, i.e., a function $f \in \Sigma$ is in $\Sigma^{*}$ if and only if

$$
\operatorname{Re}\left\{\frac{z \tilde{f}^{\prime}(z)}{\bar{f}(z)}\right\}>0
$$

for all $z \in V$.

$S^{-1}$ will be the class of functions

$$
\phi(w)=w+b_{2} w^{2}+b_{3} w^{3}+\cdots
$$

which are inverse to functions in $S$.

Presented to the Society, September 3, 1965; received by the editors May 20, 1965.

(1) This research was supported by the National Science Foundation, under research grant NSF-G 24469 with the University of Maryland. The paper represents part of the writer's Ph.D. thesis written at the University of Maryland under the direction of Professor J. A. Hummel. 
$\Sigma^{-1}$ will be the class of functions

$$
\tilde{\phi}(w)=w+\tilde{b}_{0}+\frac{\tilde{b}_{1}}{w}+\frac{\tilde{b}_{2}}{w^{2}}+\cdots
$$

which are inverse to functions in $\Sigma$.

Likewise, $S^{*^{-1}}$ and $\Sigma^{*-1}$ will denote the inverse classes of $S^{*}$ and $\Sigma^{*}$ respectively.

For the positive integer $p$ let

and let

$$
S_{p}=\left\{f_{p} \in S: f_{p}(z)=\sum_{v=0}^{\infty} a_{v p+1} z^{v p+1}\right\}
$$

$$
\Sigma_{p}=\left\{f_{p} \in \Sigma: f_{p}(z)=\sum_{v=0}^{\infty} \tilde{a}_{-1+v p} z^{-1+v p}\right\} .
$$

Finally, let $S_{p}^{*}, \Sigma_{p}^{*}, S_{p}^{-1}, \Sigma_{p}^{-1}, S_{p}^{*-1}$ and $\Sigma_{p}^{*-1}$ have the obvious meanings.

It was conjectured in 1916 by L. Bieberbach [1] that if $f \in S$ then $\left|a_{n}\right| \leqq n$, $n=2,3, \cdots$, with equality only for $f(z)=z /(1-\lambda z)^{2},|\lambda|=1$; he was able to prove this for $n=2$. In 1923, Karl Lowner [2] proved that $\left|a_{3}\right| \leqq 3$ and recently [2], [3], [6] proofs have been given for $n=4$, but the conjecture remains unverified in general. On the other hand, R. Nevanlinna [13] proved in 1921 that if $f \in S^{*}$ then $\left|a_{n}\right| \leqq n, n=2,3, \cdots$.

Lowner also proved, in his 1923 paper mentioned above, that if $\phi \in S^{-1}$ then

$$
\left|b_{n}\right| \leqq \frac{1}{n}\left(\begin{array}{c}
2 n \\
n-1
\end{array}\right) \text {. }
$$

Since the extremal function is the inverse of $f(z)=z /(1-\lambda z)^{2},|\lambda|=1$, and this is a starlike function, it follows that this is also a sharp inequality for the coefficients of the power series expansion of $\phi \in S^{*-1}$.

Until recently there were no general results for the coefficient extremal problems for functions $\tilde{f}^{*} \in \Sigma^{*}$ and $\tilde{\phi} \in \Sigma^{*-1}$. Then in $1958 \mathrm{~J}$. Clunie [4] proved that if $f^{*} \in \Sigma^{*}$ then $\left|\tilde{a}_{n}\right| \leqq 2 /(n+1), n=1,2, \cdots$, with equality only if

$$
f^{\prime}(z)=z\left(1+\lambda z^{-(n+1)}\right)^{2 /(n+1)}, \quad|\lambda|=1 .
$$

In 1963 W. C. Royster [15] conjectured that if $\tilde{\phi} \in \Sigma^{*-1}$ then

$$
\left|\tilde{b}_{n}\right| \leqq \frac{1}{n}\left(\begin{array}{c}
2 n \\
n+1
\end{array}\right), \quad n=1,2, \cdots
$$

with equality only for the inverse of $f^{\prime}(z)=z\left(1+\lambda z^{-1}\right)^{2},|\lambda|=1$, and E. Netanyahu [12] has recently presented a proof of this for $\phi \in \Sigma^{-1}$ and thus for $\phi \in \Sigma^{*-1}$.

By using very simple methods we are able to prove in this paper that if $\tilde{\phi} \in \Sigma^{*-1}$ then

$$
\left|\tilde{b}_{n}\right| \leqq \frac{1}{n}\left(\begin{array}{c}
2 n \\
n+1
\end{array}\right),
$$

while at the same time proving that if $\phi \in S^{*-1}$ then . 


$$
\left|b_{n}\right| \leqq \frac{1}{n}\left(\begin{array}{c}
2 n \\
n-1
\end{array}\right)
$$

Furthermore, by applying the variational techniques of Schiffer [24] and Schaeffer and Spencer [19], we will simultaneously prove these same inequalities for $\Sigma^{-1}$ and $S^{-1}$.

In §II the basic method used in this paper for obtaining coefficient bounds will be presented. §III will be devoted to applying the results of $\S$ II to the classes $S^{*^{-1}}$ and $\Sigma^{*-1} ; \S I V$ will be devoted to the classes $S^{-1}$ and $\Sigma^{-1}$.

It is hoped that this paper will help to unify and generalize the coefficient extremal problems for functions in the classes $S$ and $\Sigma$.

II. A coefficient matrix. In the introduction this paper was described as dealing with certain coefficient extremal problems. Specifically, we wish to consider infinite coefficient matrices the rows of which are the coefficients of the integral powers of functions in $S$ or $S^{*}$. As far as possible we will find sharp bounds for the coefficients in these matrices and use these results to find the extremal coefficients of functions in the classes $S^{-1}\left(S^{*-1}\right)$ and $\Sigma^{-1}\left(\Sigma^{*-1}\right)$. In this section we will indicate the method by which this is to be accomplished.

Definition 2.1. Let $\Omega$ be the class of functions $f$ for which there is a $\rho>0$ such that for $|z|<\rho$

$$
f(z)=\sum_{v=1}^{\infty} a_{v} z^{v}, \quad a_{1} \neq 0 .
$$

Definition 2.2. For $f \in \Omega$ we define the coefficients $a_{v}^{(t)},-\infty<t, v<\infty$, $t$ and $v$ integral, to be those such that for $|z|<\rho^{\prime}, \rho^{\prime}>0$,

$$
f(z)^{t}=\sum_{v=-\infty}^{\infty} a_{v}^{(t)} z^{v}
$$

Thus $f$ defines an infinite matrix $F=\left[a_{v}^{(t)}\right]$ and $F$ represents the function $f$.

Note that $a_{v}^{(t)}=0$ for $v<t$.

The following general results are due to Eri Jabotinsky [9].

THEOREM 2.1 (JABOTINSKY). Let $f, g \in \Omega$ and consider $k(z)=f(g(z))$ which is also in $\Omega$. Let $F=\left[a_{v}^{(t)}\right], G=\left[b_{v}^{(t)}\right]$, and $K=\left[c_{v}^{(t)}\right]$ be the matrices representing $f, g$, and $k$ respectively. Then $K=F \times G$, i.e.,

$$
c_{v}^{(t)}=\sum_{p=-\infty}^{\infty} a_{p}^{(t)} b_{v}^{(p)}
$$

THEOREM 2.2 (JABOTINSKY). If $f \in \Omega$ and $\phi$ is the inverse function of $f$, then $\phi \in \Omega$, and if

then

$$
\phi(w)^{t}=\sum_{v=-\infty}^{\infty} b_{v}^{(t)} w^{t}
$$




$$
b_{v}^{(t)}=\frac{t}{v} a_{-t}^{(-v)}, \quad v \neq 0
$$

For $v=0, b_{0}^{(t)}$ is defined by

$$
\sum_{t=-\infty}^{\infty} b_{0}^{(t)} z^{-t-1}=\frac{f^{\prime}(z)}{f(z)} .
$$

Consider any function $f \in S\left(S^{*}\right)$ : this function defines a coefficient matrix $\left[a_{v}^{(t)}\right],-\infty<t, v<\infty$, and by Theorem 2.2 if $\phi$ is the inverse of $f$, then

$$
\phi(w)=\sum_{t=1}^{\infty} \frac{1}{t} a_{-1}^{(t)} w^{t}=\sum_{t=1}^{\infty} b_{t} w^{t}
$$

and, in fact, we see that

$$
\operatorname{lmax}_{\phi \in S^{-1}\left(S^{*-1}\right)}\left|b_{n}\right|=\frac{1}{n} \max _{f \in S\left(S^{*}\right)}\left|a_{-1}^{(-n)}\right| .
$$

Since it is known that

$$
\max _{\phi \in S^{-1}\left(S^{*-1}\right)}\left|b_{n}\right|=\frac{1}{n}\left(\begin{array}{c}
2 n \\
n-1
\end{array}\right)
$$

we immediately have that

$$
\max _{f \in S\left(S^{*}\right)}\left|a_{-1}^{(-n)}\right|=\left(\begin{array}{c}
2 n \\
n-1
\end{array}\right) .
$$

However, we do not wish to use (4) in this direction, but rather in the opposite direction: if $\phi(w)=w+b_{2} w^{2}+\cdots \in S^{-1}\left(S^{*-1}\right)$, then by (4) we have

$$
\left|b_{n}\right| \leqq \frac{1}{n}\left(\max _{f \in S\left(S^{*}\right)}\left|a_{-1}^{(-n)}\right|\right)
$$

and we could thus obtain sharp bounds for $\left|b_{n}\right|$ by finding

$$
\max _{f \in S\left(S^{*}\right)}\left|a_{-1}^{(-n)}\right|
$$

This approach may be extended.

THEOREM 2.3.

$$
\max _{f \in S\left(S^{*}\right)}\left|a_{n}^{(-1)}\right|=\max _{\tilde{f} \in \Sigma\left(\Sigma^{*}\right)}\left|\tilde{a}_{n}\right|
$$

Proof. First we note that the $n$th coefficient of the expansion of $f(z)^{-1}$ about zero is the same as the $n$th coefficient of the expansion of $f(1 / z)^{-1}$ about infinity.

For every function $\tilde{f}(z) \in \Sigma$ there is a function $f(z) \in S$ such that

$$
f(1 / z)^{-1}=f(z) \text {, }
$$

so 


$$
\max _{f \in S\left(S^{*}\right)}\left|a_{n}^{(-1)}\right| \geqq \max _{f \in \Sigma\left(\Sigma^{*}\right)}\left|\tilde{a}_{n}\right|,
$$

and for every $f(z) \in S, f(1 / z)^{-1} \in \Sigma$, so that

$$
\max _{f \in S\left(S^{*}\right)}\left|a_{n}^{(-1)}\right| \leqq \max _{\tilde{f}^{*} \in \Sigma\left(\Sigma^{*}\right)}\left|\tilde{a}_{n}\right| \text {. }
$$

The theorem is thus proved for the schlicht case.

That starlikeness is preserved follows from

$$
\frac{z f^{\prime}(z)}{f(z)}=\frac{z[1 / f(1 / z)]^{\prime}}{1 / f(1 / z)}=\frac{(1 / z) f^{\prime}(1 / z)}{f(1 / z)} .
$$

This theorem is a simple and obvious consequence of the fact that there is a natural one-to-one mapping of $S\left(S^{*}\right)$ onto $\Sigma\left(\Sigma^{*}\right)$ given by $\eta: f(z) \rightarrow f(1 / z)^{-1}=\tilde{f}^{\prime}(z)$. Quite naturally the one-to-one correspondence between $S\left(S^{*}\right)$ and $\Sigma\left(\Sigma^{*}\right)$ implies a one-to-one correspondence between $S^{-1}\left(S^{*-1}\right)$ and $\Sigma^{-1}\left(\Sigma^{*-1}\right)$.

THEOREM 2.4. Let $\phi$ be the inverse function of $f$. Then $\phi(1 / w)^{-1}=\tilde{\phi}(w)$ is the inverse function of $f(1 / z)^{-1}=\tilde{f}(z)$.

Proof. $\phi$ maps $f(U)=D$ onto $U$ and so $\tilde{\phi}$ maps $\tilde{D}=\{w: 1 / w \in D\}$ onto $V$. $\tilde{f}(z)=f(1 / z)^{-1}$ maps $V$ onto $\tilde{D}$ and $\tilde{\phi}(\tilde{f}(z))=[\phi(1 / \tilde{f}(z))]^{-1}=[\phi(f(1 / z))]^{-1}$ $=(1 / z)^{-1}=z$, i.e., $\tilde{\phi}$ is indeed the inverse of $\tilde{f}$.

\section{Corollary 2.1.}

$$
\max _{\phi \in S^{-1}\left(S^{*-1}\right)}\left|b_{n}^{(-1)}\right|=\max _{\phi \in \Sigma^{-1}\left(\Sigma^{*-1}\right)}\left|\tilde{b}_{n}\right| .
$$

Proof. By Theorem 2.4, for every $\tilde{\phi}(w)$ there is $\phi(w)$ such that $\phi(1 / w)^{-1}=\tilde{\phi}(w)$ and since the coefficients in the power series expansions of $\phi(w)^{-1}$ and $\phi(1 / w)^{-1}$ are the same, we have

$$
\max _{\phi \in S^{-1}\left(S^{*-1}\right)}\left|b_{n}^{(-1)}\right| \geqq \max _{\phi^{1} \in \Sigma^{-1}\left(\Sigma^{*-1}\right)}\left|\tilde{b}_{n}\right| .
$$

Also, for $\phi(w) \in S^{-1}, \phi(1 / w)^{-1} \in \Sigma^{-1}$ so that

$$
\max _{\phi \in S^{-1}\left(S^{*-1}\right)}\left|b_{n}^{(-1)}\right| \leqq \max _{\phi \in \Sigma^{-1}\left(\Sigma^{*-1}\right)}\left|\tilde{b}_{n}\right| .
$$

If $\phi \in S^{*-1}$, then $\phi(1 / w)^{-1} \in \Sigma^{*-1}$, and if $\tilde{\phi} \in \Sigma^{*-1}$, then the $\phi$ for which $\tilde{\phi}(w)=\phi(1 / w)^{-1}$ is in $S^{*-1}$.

THEOREM 2.5 .

$$
\begin{aligned}
& \max _{\phi \in \Sigma^{-1}\left(\Sigma^{*-1}\right)}\left|\tilde{b}_{n}\right|=\frac{1}{n !} \max _{f \in S\left(S^{*}\right)}\left|a_{1}^{(-n)}\right|, \quad n=1,2, \cdots ; \\
& \max _{\phi \in \Sigma^{-1}\left(\Sigma^{*-1}\right)}\left|\tilde{b}_{0}\right|=\max _{\phi \in S^{-1}\left(S^{*-1}\right)}\left|b_{0}^{(-1)}\right| .
\end{aligned}
$$


Proof. This follows directly from Corollary 2.1 in view of Theorem 2.2.

III. Coefficient problems for $S^{*}$ and $S_{p}{ }^{*}$. Let us agree henceforth that if $f \in S\left(S^{*}\right)$ then $f(z)^{t}=\sum_{v=t}^{\infty} a_{v}^{(t)} z^{v}$ and if we write $a_{n}^{(t)}$ then this is the $n$th coefficient of the $t$ th power of some function $f \in S\left(S^{*}\right)$. Likewise if $\phi \in S^{-1}\left(S^{*-1}\right)$, then

$$
\phi(z)^{t}=\sum_{v=t}^{\infty} b_{v}^{(t)} z^{v}
$$

and if we write $b_{n}^{(t)}$, then this is the $n$th coefficient of the $t$ th power of some function $\phi \in S^{-1}\left(S^{*-1}\right)$. Further we should agree that if we write $\tilde{a}_{n}$ or $\tilde{b}_{n}$ then these are the coefficients of the series development of $\tilde{f} \in \Sigma\left(\Sigma^{*}\right)$ or $\tilde{\phi} \in \Sigma^{-1}\left(\Sigma^{*-1}\right)$.

The first part of this section (i.e., III.A.) is devoted to the problem of bounding $\left|a_{n}^{(t)}\right|,-\infty<t, n<\infty$, when $f$ is in $S^{*}$; we call the matrix of sharp bounds the maximum coefficient matrix for $S^{*}$. In III.B the generalizations (for $S_{p}$ ) of the theorems stated and used in III.A are proved.

III.A. A partial maximum coefficient matrix for $S^{*}$. Theorems 3.1 and 3.2 are special cases (for $p=1$ ) of Theorems 3.7 and 3.8 of III.B.

TheOREM 3.1. Let $f \in S^{*}$. For $t>0$

$$
\left|a_{t+n}^{(t)}\right| \leqq\left|\left(\begin{array}{c}
-2 t \\
n
\end{array}\right)\right|, \quad n=1,2, \cdots,
$$

and this inequality is sharp.

If $t=1$, then (7) becomes

$$
\left|a_{1+n}\right| \leqq\left|\left(\begin{array}{c}
-2 \\
n
\end{array}\right)\right|=\left|\frac{(-2)(-3) \cdots(-n-1)}{n \cdots 1}\right|=n+1,
$$

a well-known inequality.

THEOREM 3.2. Let $f \in S^{*}$. For $t>0$

$$
\left|a_{-t+n}^{(-t)}\right| \leqq\left(\begin{array}{c}
2 t \\
n
\end{array}\right), \quad n=1,2, \cdots t+1,
$$

and this inequality is sharp.

In §II we thoroughly discussed the correspondence between the coefficients of the power series developments of $\phi \in S^{-1}\left(S^{*-1}\right)$ and $\tilde{\phi} \in \Sigma^{-1}\left(\Sigma^{*-1}\right)$ and the coefficients of the series representations of the powers of the functions $f \in S\left(S^{*}\right)$. The usefulness of this is now evident.

THEOREM 3.3. If $\phi \in S^{*-1}$, then we have the sharp estimate

$$
\left|b_{n}\right| \leqq \frac{1}{n}\left(\begin{array}{c}
2 n \\
n-1
\end{array}\right)
$$


Proof. By equation (4) and Theorem 3.2

$$
\left|b_{n}\right| \leqq \frac{1}{n} \max _{f \in S^{*}}\left|a_{-1}^{(-n)}\right|-\frac{1}{n}\left(\begin{array}{c}
2 n \\
n-1
\end{array}\right) .
$$

Since $\left(\begin{array}{c}2 n \\ n+1\end{array}\right)$ is a sharp bound for $\left|a_{-1}^{(-n)}\right|,(9)$ is sharp.

THEOREM 3.4. If $\tilde{\phi} \in \Sigma^{*-1}$, then we have the sharp estimate

$$
\left|\tilde{b}_{n}\right| \leqq \frac{1}{n}\left(\begin{array}{c}
2 n \\
n+1
\end{array}\right), \quad n=1,2, \cdots,
$$

$$
\left|\tilde{b}_{0}\right| \leqq 2 \text {. }
$$

Proof. By equation (6) and Theorem 3.2

$$
\left|\tilde{b}_{n}\right| \leqq \frac{1}{n} \max _{f \in S^{*}}\left|a_{1}^{(-n)}\right|=\frac{1}{n}\left(\begin{array}{c}
2 n \\
n+1
\end{array}\right) .
$$

To see that $\left|\tilde{b}_{0}\right| \leqq 2$ we consider equation (3):

$$
\begin{aligned}
\sum_{t=-\infty}^{\infty} b_{0}^{(t)} z^{-t-1} & =\frac{1}{z} \frac{z f^{\prime}(z)}{f(z)} \\
& =\frac{1}{z} g(z) \\
& =\frac{1}{z}\left(1+\sum_{v=1}^{\infty} c_{v} z^{v}\right),
\end{aligned}
$$

where $\left|c_{v}\right| \leqq 2$. Hence $\left|b_{0}^{(-n)}\right| \leqq 2$ for $n>0$. In particular, by equation (6),

$$
\left|\tilde{b}_{0}\right| \leqq \max _{\phi \in S^{*-1}}\left|b_{0}^{(-1)}\right| \leqq 2
$$

Since $\left(\begin{array}{c}2 n \\ n+1\end{array}\right)$ is a sharp bound for $\left|a_{1}^{(-n)}\right|$ and 2 is a sharp bound for $\left|b_{0}^{(-1)}\right|$ the inequalities (10) are sharp.

REMARK 3.1. It should be noted here that we have proved more - that $\left|b_{0}^{(-n)}\right| \leqq 2, n>0,-$ while proving the last theorem.

We see in III.B that Theorem 3.2 is proved by a generalization of a method due to Clunie [4].

In the next section we prove Theorem 3.2 for the class $S$ and hence will have Theorems 3.3 and 3.4 for $S^{-1}$ and $\Sigma^{-1}$ but in order to accomplish this we will have to use variational methods. Here, though, we have been able to obtain these theorems for $S^{*}$ by using relatively simple techniques. Theorem 3.3 has also been proved by variational methods [18] as well as by Lowner [10] and another proof of Theorem 3.4 exists (Netanyahu [12]), but here we have, very likely, the simplest proofs of these theorems.

For the sake of completeness we include Clunie's result concerning $\Sigma^{*}$ [4]. 
Theorem 3.5 (J. Clunie). If $\tilde{f} \in \Sigma^{*}$, then

$$
\left|\tilde{a}_{n}\right| \leqq \frac{2}{n+1}, \quad n=0,1,2, \cdots,
$$

with equality for $f(z)=z\left(1+z^{(n+1)}\right)^{2 /(n+1)}$.

Figure 1 summarizes Theorems 3.1, 3.2, and 3.5. If $n>t$ then we can write (7) as

$$
\left|a_{n}^{(t)}\right| \leqq\left|\left(\begin{array}{c}
-2 t \\
n-t
\end{array}\right)\right|
$$

so in Figure 1 we assume $n>t$ and use this form of (7).

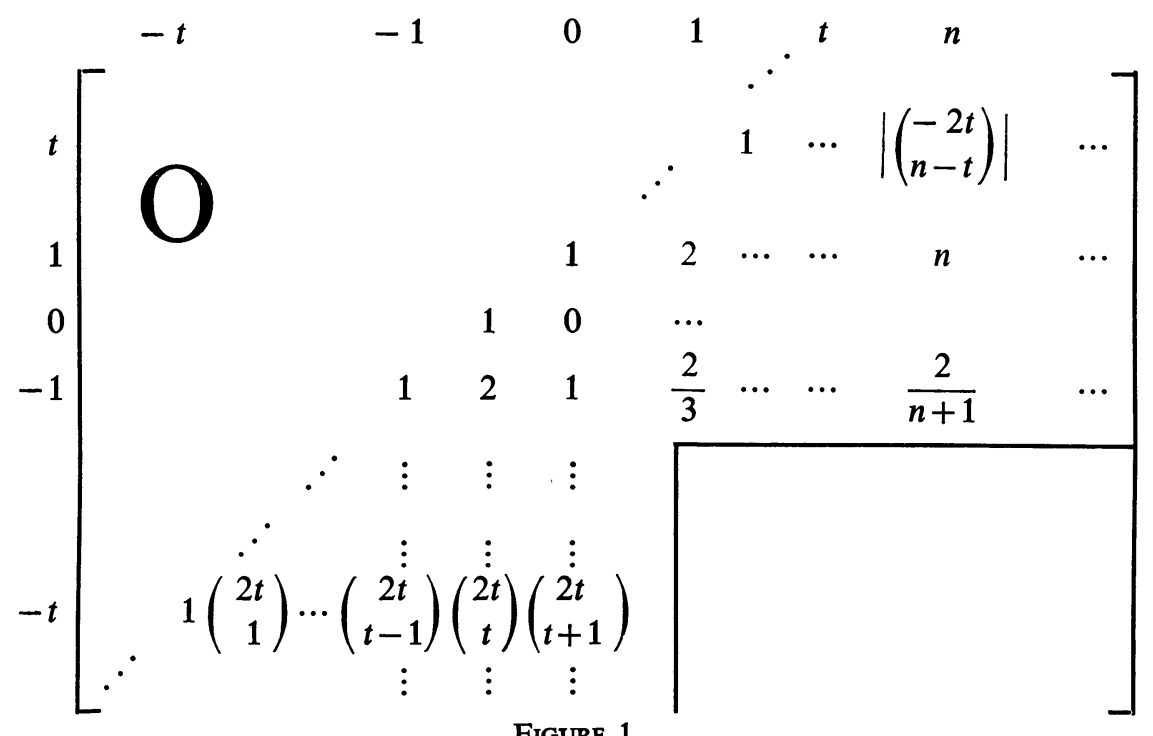

FIGURE 1

Maximum Coefficient Matrix for $S^{*}$

We now move on to investigate the region $t\langle-1, n\rangle 1$, of the maximum coefficient matrix for $S^{*}$. In order to do this we will use Prawitz's [14] generalization of the Area Theorem.

Theorem 3.6 (Prawitz). If $f \in S\left(S^{*}\right)$ and $t>0$, then

$$
\sum_{v=1}^{\infty} v\left|a_{v}^{(-t)}\right|^{2} \leqq t+(t-1)\left|a_{-t+1}^{(-t)}\right|^{2}+\cdots+\left|a_{-1}^{(-t)}\right|^{2} .
$$

In view of Theorem 3.2 we have the following corollaries to Theorem 3.6.

Corollary 3.1. If $f \in S^{*}$ and $t>0$, then

$$
\sum_{v=1}^{\infty} v\left|a_{v}^{(-t)}\right|^{2} \leqq \frac{t}{4}\left(\begin{array}{c}
2 t \\
t
\end{array}\right)^{2}
$$


Proof. The right-hand side of (12) is

$$
\begin{aligned}
t+(t-1) & \left|a_{-t+1}^{(-t)}\right|^{2}+\cdots+\left|a_{-1}^{(-t)}\right|^{2} \\
& \leqq t+(t-1)\left(\begin{array}{c}
2 t \\
1
\end{array}\right)+\cdots+\left(\begin{array}{c}
2 t \\
t-1
\end{array}\right)^{2} \\
& =\frac{t}{4}\left(\begin{array}{c}
2 t \\
t
\end{array}\right)^{2} .
\end{aligned}
$$

COROllary 3.2. If $f \in S^{*}$ and $t>0$, then

$$
\sum_{v=1}^{t} v\left|a_{v}^{(-t)}\right|^{2} \leqq \frac{t}{4}\left(\begin{array}{c}
2 t \\
t
\end{array}\right)^{2}
$$

and this inequality is sharp as $\left(z /(1+z)^{2}\right)^{-t}$ shows. If equality holds, then $a_{n}^{(-t)}=0$ for $n>t$.

Proof. The inequality follows immediately from Corollary 3.1. For the function $\left(z /(1+z)^{2}\right)^{-t}$ the sum

$$
\sum_{v=1}^{t}\left|a_{v}^{(-t)}\right|^{2}=\sum_{v=1}^{t}\left(\begin{array}{c}
2 t \\
t+v
\end{array}\right)^{2}=\frac{t}{4}\left(\begin{array}{c}
2 t \\
t
\end{array}\right)^{2}
$$

showing that (13) is sharp. Now it obviously follows from Corollary 3.1 that if equality holds then $a_{n}^{(-t)}$ must be zero if $n>t$.

III.B. $S_{p}^{*}$.

THEOREM 3.7. If $f \in S^{*}$, then for integral $p>0, f\left(z^{p}\right)^{1 / p} \in S^{*}$, and for $t>0$ we have the sharp inequality

where

$$
\left|\begin{array}{c}
(t / p) \\
t+n p
\end{array}\right| \leqq\left|\left(\begin{array}{c}
-2 t / p \\
n
\end{array}\right)\right|, \quad n=1,2, \cdots,
$$

$$
f\left(z^{p}\right)^{t / p}=\sum_{v=0}^{\infty} a_{t+v p}^{(t / p)} z^{t+v p}, \quad a_{t}^{(t / p)}=1
$$

Proof. For $f \in S^{*}$

$$
\begin{aligned}
\frac{z\left[f\left(z^{p}\right)^{1 / p}\right]^{\prime}}{f\left(z^{p}\right)^{1 / p}} & =\frac{z f\left(z^{p}\right)^{1 / p-1} f^{\prime}\left(z^{p}\right) z^{p-1}}{f\left(z^{p}\right)^{1 / p}} \\
& =z^{p} \frac{f^{\prime}\left(z^{p}\right)}{f\left(z^{p}\right)}
\end{aligned}
$$

and therefore, for $z \in U$,

$$
\operatorname{Re}\left\{\frac{z\left[f\left(z^{p}\right)^{1 / p}\right]^{\prime}}{f\left(z^{p}\right)^{1 / p}}\right\}=\operatorname{Re}\left\{z^{p} \frac{f^{\prime}\left(z^{p}\right)}{f\left(z^{p}\right)}\right\}>0 .
$$


Hence $f\left(z^{p}\right)^{1 / p} \in S^{*}$.

Now we have

$$
\frac{1}{t} z \frac{\left[f\left(z^{p}\right)^{t / p}\right]^{\prime}}{f\left(z^{p}\right)^{t / p}}=z^{p} \frac{f^{\prime}\left(z^{p}\right)}{f\left(z^{p}\right)}=g\left(z^{p}\right)=1+\sum_{v=1}^{\infty} c_{v p} z^{v p},
$$

where $\left|c_{v p}\right| \leqq 2, v=1,2, \cdots$, and since

we obtain

$$
f\left(z^{p}\right)^{t / p}=\sum_{v=0}^{\infty} a_{t+v p}^{(t / p)} z^{t+v p}, \quad a_{t}^{(t / p)}=1
$$

$$
\sum_{v=0}^{\infty}(t+v p) a_{t+v p}^{(t / p)} a^{t+v p}=t\left(\sum_{v=0}^{\infty} a_{t+v p}^{(t / p)} z^{t+v p}\right)\left(1+\sum_{v=1}^{\infty} c_{v p} z^{v p}\right) .
$$

Comparing coefficients:

$$
(n p+t) a_{n p+t}^{(t / p)}=t\left[a_{n p+t}^{(t / p)}+c_{p} a_{(n-1) p+t}^{(t / p)}+\cdots+c_{n p}\right]
$$

or

$$
n p a_{n p+t}^{(t / p)}=t\left[c_{p} a_{(n-1) p+t}^{(t / p)}+\cdots+c_{n p}\right]
$$

For $n=1$,

Assume

$$
\left|a_{p+t}^{(t / p)}\right|=\frac{t\left|c_{p}\right|}{p} \leqq \frac{2 t}{p}=\left|\left(\begin{array}{c}
-2 t / p \\
1
\end{array}\right)\right|
$$

Then

$$
\left|a_{v p+t}^{(t / p)}\right| \leqq\left|\left(\begin{array}{c}
-2 t / p \\
v
\end{array}\right)\right|, v=1,2, \cdots, n-1
$$

$$
\begin{aligned}
\left|a_{n p+t}^{(t / p)}\right| & \leqq \frac{2 t}{n p}\left[\left|a_{(n-1) p+t}^{(t / p)}\right|+\cdots+1\right] \\
& \leqq \frac{2 t}{n p}\left[\left|\left(\begin{array}{c}
-2 t / p \\
n-1
\end{array}\right)\right|+\cdots+1\right] \\
& =\left|\left(\begin{array}{c}
-2 t / p \\
n
\end{array}\right)\right| .
\end{aligned}
$$

Thus, by induction we get the desired result.

This inequality is sharp:

$$
\left(\frac{z}{\left(1+z^{p}\right)^{2 / p}}\right)^{t}=z^{t}\left(1+\sum_{v=1}^{\infty}\left(\begin{array}{c}
-2 t / p \\
v
\end{array}\right) z^{v p}\right) .
$$

REMARK 3.2. For every integral $p>0$ the function

may be expressed as

$$
f_{p}(z)=\sum_{v=0}^{\infty} a_{v p+1} z^{v p+1} \in S_{p}^{*}
$$




$$
f_{p}(z)=f\left(z^{p}\right)^{1 / p}
$$

for some $f(z) \sum_{v=1}^{\infty} a_{v} z^{v} \in S^{*}$ and for every $f \in S^{*}, f\left(z^{p}\right)^{1 / p} \in S_{p}^{*}$. Thus when we consider the extremal coefficient problem for functions $f_{p}(z) \in S_{p}^{*}$, we may deal instead with functions $f\left(z^{p}\right)^{1 / p}$ for $f \in S^{*}$.

Corollary 3.3. If $f_{p} \in S_{p}^{*}$ and $t>0$, then we have the sharp estimate

$$
\left|a_{n p+t}^{(t)}\right| \leqq\left|\left(\begin{array}{c}
-2 t / p \\
n
\end{array}\right)\right|, \quad n=1,2, \cdots .
$$

Proof. This is just a restatement of Theorem 3.7 in view of Remark 3.2.

THEOREM 3.8. If $f \in S^{*}$, then for $t>0, p>0$,

$$
\left|a_{-t+n / p}^{(-t / p)}\right| \leqq\left(\begin{array}{c}
2 t / p \\
n
\end{array}\right)
$$

for all $n$ such that $p(n-1)-t \leqq 0,(c f$. Theorem 3.2), and for $t \leqq p$

$$
\left|a_{-t+n p}^{(-t / p)}\right| \leqq \frac{2 t}{n p}, \quad n=1,2, \cdots
$$

(cf. Theorem 3.5), where $f\left(z^{p}\right)^{-t / p}=\sum_{v=0}^{\infty} a_{-t+v p}^{(-t / p)} z^{-t+v p}, a_{-t}^{(-t / p)}=1$.

Proof. For $f \in S^{*}$

$$
\frac{1}{t} \frac{z\left[f\left(z^{p}\right)^{-t / p}\right]^{\prime}}{f\left(z^{p}\right)^{-t / p}}=\frac{z^{p} f^{\prime}\left(z^{p}\right)}{f\left(z^{p}\right)}=g\left(z^{p}\right) .
$$

We define

$$
w\left(z^{p}\right)=\frac{g\left(z^{p}\right)-1}{g\left(z^{p}\right)+1}=\sum_{v=1}^{\infty} \omega_{v p} z^{v p}
$$

Thus

$$
\frac{z\left[f\left(z^{p}\right)^{-t / p}\right]^{\prime}+t f\left(z^{p}\right)^{-t / p}}{z\left[f\left(z^{p}\right)^{-t / p}\right]^{\prime}-t f\left(z^{p}\right)^{-t / p}}=\sum_{v=1}^{\infty} \omega_{v p} z^{v p}
$$

and since $f\left(z^{p}\right)^{-t / p}=\sum_{v=0}^{\infty} a_{-t+v p}^{(-t / p)} z^{-t+v p}, a_{-t}^{(-t / p)}=1$, we have

$$
\sum_{v=1}^{\infty} v p a_{-t+v p}^{(-t / p)} z^{-t+v p}=\left(-2 t z^{-t}+\sum_{v=1}^{\infty}(-2 t+v p) a_{-t+v p}^{(-t / p)} z^{-t+v p}\right)\left(\sum_{v=1}^{\infty} \omega_{v p} z^{v p}\right) .
$$

Thus

$$
\begin{gathered}
\left(-2 t z^{-t}+\sum_{v=1}^{n-1}(-2 t+v p) a_{-t+v p}^{(-t / p)} z^{-t+v p}\right)\left(\sum_{v=1}^{n-1} \omega_{v p} z^{v p}\right) \\
=\sum_{v=1}^{n} v p a_{-t+v p}^{(-t / p)} z^{-t+v p}+\sum_{v=n+1}^{\infty} c_{v} z^{-t+v p} .
\end{gathered}
$$


Now multiplying each side by its conjugate, integrating around $|z|=r<1$ (noting that $\left|w\left(z^{p}\right)\right|<1$ ), letting $r \rightarrow 1$, and then discarding $\sum_{v=n+1}^{\infty}\left|c_{v}\right|^{2}$, we obtain

$$
\sum_{v=1}^{n}(v p)^{2}\left|a_{-t+v p}^{(-t / p)}\right|^{2} \leqq 4 t^{2}+\sum_{v=1}^{n-1}(-2 t+v p)^{2}\left|a_{-t+v p}^{(-t / p)}\right|^{2},
$$

from which

$$
(n p)^{2}\left|a_{-t+n p}^{(-t / p)}\right|^{2} \leqq 4 t^{2}+\sum_{v=1}^{n-1}\left(4 t^{2}-4 t v p\right)\left|a_{-t+v p}^{(-t / p)}\right|^{2}
$$

$$
=4 t\left[t+\sum_{v=1}^{n-1}(t-v p)\left|a_{-t-v p}^{(-t / p)}\right|^{2}\right] .
$$

If $t \leqq p$, this gives

$$
\begin{aligned}
(n p)^{2}\left|a_{-t+n p}^{(-t / p)}\right|^{2} & \leqq 4 t^{2}, \\
\left|a_{-t+n p}^{(-t / p)}\right| & \leqq \frac{2 t}{n p}, \quad n=1,2, \cdots .
\end{aligned}
$$

For $t>p$ we have

$$
\left|a_{-t+p}^{(-t / p)}\right| \leqq \frac{2 t}{p}
$$

and assuming

$$
\left|a_{-t+v p}^{(-t / p)}\right| \leqq\left(\begin{array}{c}
2 t / p \\
v
\end{array}\right), \quad v=1, \cdots, n-1,
$$

where $p(n-1)-t \leqq 0$, we have

$$
(n p)^{2}\left|a_{-t+n p}^{(-t / p)}\right|^{2} \leqq 4 t\left[t+\sum_{v=1}^{n-1}(t-v p)\left(\begin{array}{c}
2 t / p \\
v
\end{array}\right)^{2}\right]=(n p)\left(\begin{array}{c}
2 t / p \\
n
\end{array}\right)^{2} .
$$

Thus

$$
\left|a_{-t+n p}^{(-t / p)}\right| \leqq\left(\begin{array}{c}
2 t / p \\
n
\end{array}\right), \quad p(n-1)-t \leqq 0 .
$$

These inequalities are sharp as is shown by $z^{-t}\left(1+z^{p}\right)^{2 t / p}$ and $z^{-t}\left(1+z^{n p}\right)^{2 t / n p}$.

Corollary 3.4. If $f_{p} \in S_{p}^{*}$ and $t>0$ then we have the sharp estimate

$$
\left|a_{-t+n p}^{(-t)}\right| \leqq\left(\begin{array}{c}
2 t / p \\
n
\end{array}\right)
$$

for all $n$ such that $p(n-1)-t \leqq 0$, and for $t \leqq p$ 


$$
\left|a_{-t+n p}^{(-t)}\right| \leqq \frac{2 t}{n p}, \quad n=1,2, \cdots
$$

Proof. This is just a restatement of Theorem 3.8 in view of Remark 3.2.

Figure 2 summarizes the results for $S_{p}^{*}$.

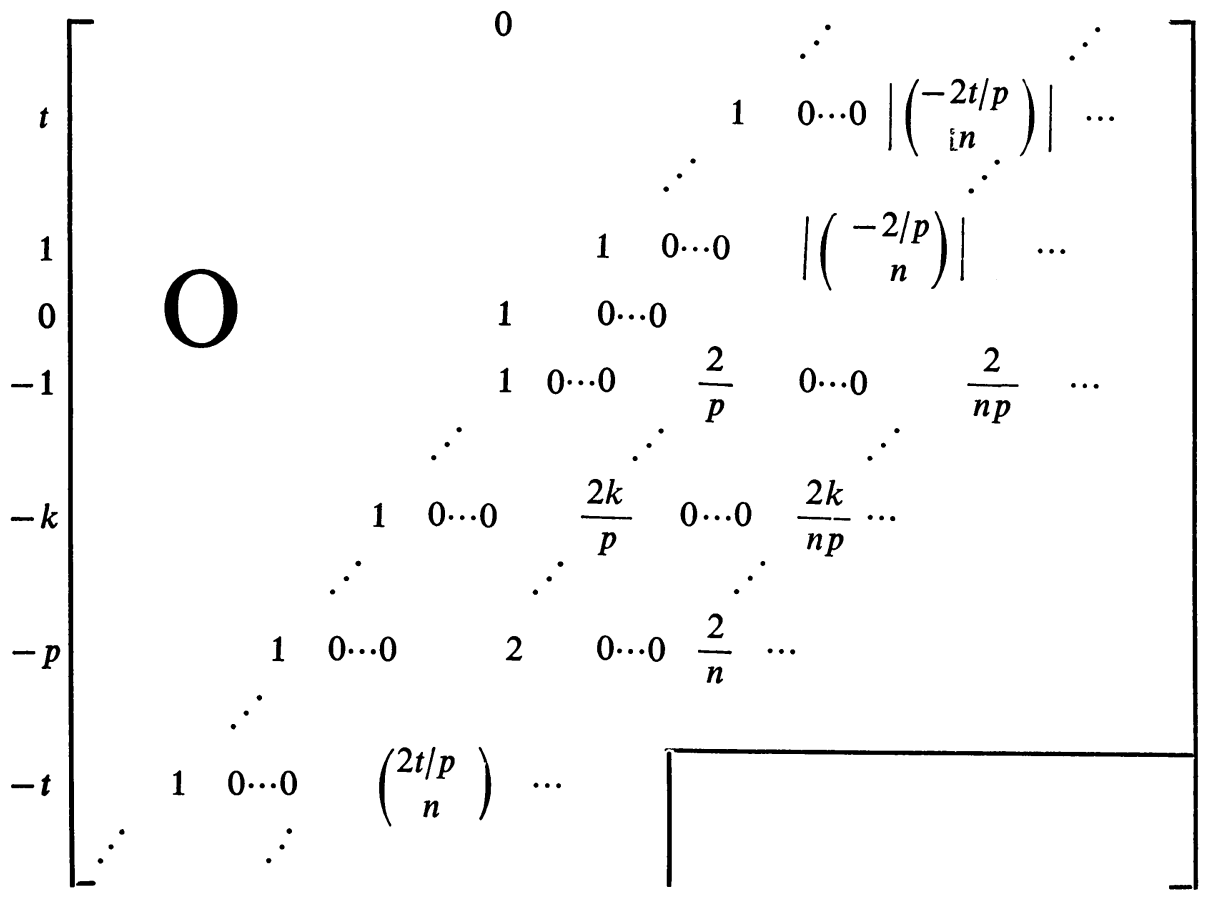

FIGURE 2

Maximum Coefficient Matrix for $S_{p}^{*}$

THEOREM 3.8. If $\phi_{p} \in S_{p}^{*-1}, \phi_{p}(w)=\sum_{v=0}^{\infty} b_{v p+1} w^{v p+1}$, then

$$
\left|b_{n p+1}\right| \leqq \frac{1}{n p+1}\left(\begin{array}{c}
2(n p+1) / p \\
n
\end{array}\right), \quad n=1,2, \cdots,
$$

and the inequality is sharp.

Proof. By (4) and (17)

$$
\begin{aligned}
\left|b_{n p+1}\right| & \leqq \frac{1}{n p+1} \max _{f \in S_{p}^{*}}\left|a_{-(n p+1)+n p}^{(-(n p+1))}\right| \\
& \leqq \frac{1}{n p+1}\left(\begin{array}{c}
2(n p+1)_{l}^{\prime} p \\
n
\end{array}\right) .
\end{aligned}
$$

The estimate is sharp since (17) is sharp. 
REMARK 3.3. If $\tilde{f}_{p} \in \Sigma_{p}^{*}$, then there is $f_{p} \in S_{p}^{*}$ such that $\tilde{f}_{p}(z)=f_{p}(1 / z)^{-1}$ and for every $f_{p} \in S_{p}^{*}, f_{p}(1 / z)^{-1} \in \Sigma_{p}^{*}$. Hence there is a natural one-to-one relation between $S_{p}^{*}$ and $\Sigma_{p}^{*}$ and thus the equations (5) and (6) of $\S$ II are valid even when we restrict out attention to $S_{p}^{*}$ and $\Sigma_{p}^{*}$.

Hence we have the following results.

THEOREM 3.9. If $\tilde{f}_{p} \in \Sigma_{p}^{*}$, then we have the sharp estimate

$$
\left|\tilde{a}_{-1+n p}\right| \leqq \frac{2}{n p}, \quad n=1,2, \cdots .
$$

Proof. This follows from (5) and (18), and the fact that (18) is sharp.

THEOREM 3.10. If $\tilde{\phi}_{p} \in \Sigma_{p}^{*-1}, \tilde{\phi}_{p}(w)=\sum_{v=0}^{\infty} \tilde{b}_{-1+v p} w^{-1+v p}$,

$$
\left|\tilde{b}_{-1+n p}\right| \leqq \frac{1}{n p-1}\left(\begin{array}{c}
2(n p-1) / p \\
n
\end{array}\right), \quad n=1,2, \cdots,
$$

and this inequality is sharp.

Proof. By (6) and (17) we have

$$
\begin{aligned}
\tilde{b}_{-1+n p} & \leqq \frac{1}{n p-1} \max _{f_{p} \in S^{p}}\left|a_{1}^{(1-n p)}\right| \\
& =\frac{1}{n p-1}\left(\begin{array}{c}
2(n p-1) / p \\
n
\end{array}\right), \quad n=1,2, \cdots .
\end{aligned}
$$

The inequality is sharp since (17) is sharp.

IV. Coefficient problems for $S$. The theory of variation of schlicht functions yields for $S$ some of the results of §III.

Variational methods for schlicht functions have been extensively used in studying coefficient extremal problems; the facts used here may be found in papers by M. Schiffer, A. C. Schaeffer, and D. C. Spencer [19]- [22], [24], [26].

Let $f \in S$. For $\varepsilon$ sufficiently small and $z_{0} \in U$ the function $f^{\Delta}$,

$$
\begin{aligned}
& f^{\Delta}(z)=f(z)+\varepsilon\left\{e ^ { i \theta } \left(-\frac{z f^{\prime}(z) f\left(z_{0}\right)}{z_{0} f^{\prime}\left(z_{0}\right)^{2}\left(z-z_{0}\right)}-\frac{f(z) f\left(z_{0}\right)}{z_{0}^{2} f^{\prime}\left(z_{0}\right)^{2}}\right.\right. \\
& \left.\left.+\frac{f(z)^{2}}{f\left(z_{0}\right)\left(f(z)-f\left(z_{0}\right)\right)}-\right)+e^{-i \theta} \frac{z^{2} f^{\prime}(z) \overline{f\left(z_{0}\right)}}{\bar{z}_{0} \overline{f^{\prime}\left(z_{0}\right)^{2}}\left(1-\bar{z}_{0} z\right)}\right\}+O\left(\varepsilon^{2}\right),
\end{aligned}
$$

is again a member of $S$ [24]. This is used to obtain a variational formula for $f\left(z^{p}\right)^{t / p}=\sum_{v=0}^{\infty} a_{t+v p}^{(t / p)} z^{t+v p}, a_{t}^{(t / p)}=1$, where $f \in s$. Let $k(z)$ represent that portion of (19) which is in braces; then $f^{\Delta}\left(z^{p}\right)^{1 / p} \in S$ and

where

$$
\begin{aligned}
f^{\Delta}\left(z^{p}\right)^{t / p} & =\left[f\left(z^{p}\right)+\varepsilon k\left(z^{p}\right)+O\left(\varepsilon^{2}\right)\right]^{t / p} \\
& =f\left(z^{p}\right)^{t / p}+\varepsilon \frac{t}{p} f\left(z^{p}\right)^{t / p-1} k\left(z^{p}\right)+O\left(\varepsilon^{2}\right),
\end{aligned}
$$




$$
\begin{aligned}
& \frac{t}{p} \varepsilon f\left(z^{p}\right)^{t / p-1} k\left(z^{p}\right) \\
&=\varepsilon\left\{e ^ { i \theta } \left(-\frac{\frac{1}{p} z\left[f\left(z^{p}\right)^{t / p}\right]^{\prime} f\left(z_{0}\right)}{z_{0} f^{\prime}\left(z_{0}\right)^{2}\left(z^{p}-z_{0}\right)}-\frac{\frac{t]}{p} f\left(z^{p}\right)^{t / p} f\left(z_{0}\right)}{z_{0} f^{\prime}\left(z_{0}\right)^{2}}\right.\right. \\
&\left.+\frac{\frac{t}{p} f\left(z^{p}\right)^{t / p+1}}{f\left(z_{0}\right)\left(f\left(z^{p}\right)-f\left(z_{0}\right)\right)}\right) \\
&\left.+e^{-i \theta} \frac{\frac{1}{p} z^{p+1}\left[f\left(z^{p}\right)^{t / p}\right]^{\prime} \overline{f\left(z_{0}\right)}}{\bar{z}_{0} \overline{f^{\prime}\left(z_{0}^{2}\right)}\left(1-\bar{z}_{0} z^{p}\right)}\right\} .
\end{aligned}
$$

Comparing coefficients we have the formula

$$
\begin{aligned}
\left.a_{t+n p}^{(t / p)}\right)_{t+n p}^{(t / p)}+\varepsilon\left\{e^{i \theta}\right. & {\left[\frac{f\left(z_{0}\right)}{z_{0}^{2} f^{\prime}\left(z_{0}\right)^{2}}\left(n a_{t+n p}^{(t / p)}+\sum_{v=0}^{n-1} \frac{(v+t / p) a_{t+v p}^{(t / p)}}{z_{0}^{n-v}}\right)\right.} \\
& \left.-\frac{t}{p} \frac{1}{f\left(z_{0}\right)} S_{t+n p}^{(t / p)}\left(\frac{1}{f\left(z_{0}\right)}\right)\right] \\
& \left.+e^{-i \theta} \frac{\overline{f\left(z_{0}\right)}}{z_{0}^{2} \overline{f^{\prime}\left(z_{0}\right)^{2}}}-\sum_{v=0}^{n-1}(v+t / p) a_{t+v p}^{(t / p)} z_{0}^{n-v}\right\}+O\left(\varepsilon^{2}\right),
\end{aligned}
$$

since

$$
\begin{aligned}
& \frac{f\left(z^{p}\right)^{t / p}}{1-\frac{1}{f\left(z_{0}\right)} f\left(z^{p}\right)}=\sum_{v=0}^{\infty}\left(a_{t+v p}^{(t / p)}+S_{t+v p}^{(t / p)}\left(\frac{1}{f\left(z_{0}\right)}\right)\right) z^{t+v p}, \\
& -\frac{\frac{1}{p} z\left[f\left(z^{p}\right)^{t / p}\right]^{\prime}}{z^{p}-z_{0}}=\frac{1}{z} \frac{1}{p} \sum_{v=0}^{\infty}(t+v p) a_{t+v p}^{(t / p)} z^{t+v p} \sum_{v=0}^{\infty} \frac{z^{v p}}{z_{0}^{v}}, \\
& \frac{1}{p} z^{p+1}\left[f\left(z^{p}\right)^{t / p}\right]^{\prime} \\
& 1-\frac{\bar{z}_{0} z^{p}}{\bar{z}_{0}}
\end{aligned}
$$

We shall use (20) to investigate the problem of maximizing $\left|a_{t+n p}^{(t / p)}\right|$ for functions $f \in S$.

It may be assumed that $a_{t+n p}^{(t / p)}$ is real and positive because if

$$
f\left(z^{p}\right)^{t / p}=z^{t}+\cdots+e^{i \theta}\left|a_{t+n p}^{(t / p)}\right| z^{t+n p}+\cdots
$$

then $e^{i \theta / n p} f\left(e^{-i \theta / n} z^{p}\right)^{1 / p} \in S$ and

$$
\left[e^{i \theta / n p} f\left(e^{-i \theta / n} z^{p}\right)^{1 / p}\right]^{t}=z^{t}+\cdots+\left|a_{i+n p}^{(t / p)}\right| z^{t+n p}+\cdots .
$$


Hence, without loss of generality, we may consider the problem of finding $\max \operatorname{Re}\left\{a_{t+n p}^{(t / p)}\right\}$ instead of finding $\max \left|a_{t+n p}^{(t / p)}\right|$ since $\max \left|a_{t+n p}^{(t / p)}\right|=\max \operatorname{Re}\left\{a_{t+n p}^{(t / p)}\right\}$.

Suppose the function $f \in S$ has $\operatorname{Re}\left\{a_{t+n p}^{(t / p)}\right\}$ maximal, i.e., $f$ is extremal for $a_{t+n p}^{(t) p}$, then for any function $f^{\Delta}\left(z^{p}\right)^{t / p}=f\left(z^{p}\right)^{t / p}+\varepsilon k\left(z^{p}\right)+O\left(\varepsilon^{2}\right)$ we have,

$$
\operatorname{Re}\left\{a_{t+n p}^{(t / p)}-a_{t+n p}^{(t / p)}\right\} \leqq 0
$$

Since (from (20))

$$
\begin{aligned}
& \operatorname{Re}\left\{a_{t+n p}^{(t / p)^{\Delta}}-a_{t+n p}^{(t / p)}\right\} \\
& =\varepsilon \operatorname{Re}\left\{e ^ { i \theta } \left[\frac { f ( z _ { 0 } ) } { z _ { 0 } ^ { 2 } f ^ { \prime } ( z _ { 0 } ) ^ { 2 } } \left(n a_{t+n p}^{(t / p)}+\sum_{v=0}^{n-1} \frac{(v+t / p) a_{t+v p}^{(t / p)}}{z_{0}^{n-v}}\right.\right.\right. \\
& \left.+\sum_{v=0}^{n-1}(v+t / p) \overline{a_{t+v p}^{(t / p)}} z_{0}^{n-v}\right) \\
& \left.\left.-\frac{t}{p} \frac{1}{f\left(z_{0}\right)} S_{t+n p}^{(t / p)}\left(\frac{1}{f\left(z_{0}\right)}\right)\right]\right\}+O\left(\varepsilon^{2}\right),
\end{aligned}
$$

where we have conjugated the term in (20) which has $e^{-i \theta}$ as a factor, and $\theta$ is arbitrary, we have that $f$ satisfies the differential equation

$$
\begin{aligned}
\frac{t}{p} \frac{z^{2} f^{\prime}(z)^{2}}{f(z)^{2}} S_{t+n p}^{(t / p)}( & \left.\frac{1}{f(z)}\right) \\
& =n a_{t+n p}^{(t / p)}+\sum_{v=0}^{n-1} \frac{(v+t / p) a_{t+v p}^{(t / p)}}{z^{n-v}}+\sum_{v=0}^{n-1}(v+t / p) \overline{a_{t+y v p}^{(t / p)}} z^{n-v} \\
& =Q(z)
\end{aligned}
$$

if it is extremal for $a_{t+n p}^{(t / p)}$.

Now if $f$ is extremal for $a_{t+n p}^{(t / p)}$, then it maps $U$ onto a domain $D$ which covers the entire $w$-plane except for analytic slits and maps $|z|=1$ onto these slits [21]. At the tips of the slits $f^{\prime}(z)$ has a zero of at least first order. Thus $f^{\prime}(z)$ has at least one zero on the unit circle, and since

$$
\frac{t}{p} \frac{z^{2} f^{\prime}(z)^{2}}{f(z)^{2}} S_{t+n p}^{(t / p)}\left(\frac{1}{f(z)}\right)=Q(z),
$$

$Q(z)$ has at least one zero on the unit circle.

We are now ready to prove the main theorem of this section and, to be sure, of the paper.

TheOREM 4.1 (CF. THeORem 3.8). If $f \in S, p, t>0, p, t$ integral, then we have the sharp inequality

$$
\left|a_{-t+n p}^{(t / p)}\right| \leqq\left(\begin{array}{c}
2 t / p \\
n
\end{array}\right)
$$

for all $n$ such that $p(n-1)-t \leqq 0$. 
Proof. By the forgoing discussion, if $f$ is extermal for $a_{-t+n p}^{(-t / p)}$ (i.e., $\operatorname{Re}\left\{a_{-t+n p}^{(-t / p)}\right\}$ is maximum for $f$ ), then there is a zero $z_{0}$ of $Q(z)$ on the unit circle. Thus

$$
\begin{aligned}
Q\left(z_{0}\right) & =n a_{-t+n p}^{(-t / p)}+\sum_{v=0}^{n-1} \frac{(v-t / p) a_{-t+v p}^{-t / p)}}{z_{0}^{n-v}}+\sum_{v=0}^{n-1}(v-t / p) \overline{a_{-t+v p}^{(-t / p)} z_{0}^{n-v}} \\
& =0
\end{aligned}
$$

so

and

$$
n a_{-t+n p}^{(-t / p)}=2 \operatorname{Re}\left\{\sum_{v=0}^{n-1}\left(\frac{t}{p}-v\right) a_{-t+v p}^{(-t / p)} z_{0}^{n-v}\right\}
$$

$$
n\left|a_{-t+n p}^{(-t / p)^{p}}\right| \leqq 2 \sum_{v=0}^{n-1}\left|\frac{t}{p}-v\right|\left|a_{-t+v p}^{(-t / p)}\right|
$$

We proceed to prove the theorem by finite induction. For $n=1$ we have

$$
\left|a_{-t+p}^{(-t / p)}\right| \leqq 2\left|\frac{t}{p}\right|=\left(\begin{array}{c}
2 t / p \\
1
\end{array}\right)
$$

Suppose that $n-1<t / p$ and assume that, for $v=1,2, \cdots, n-1$,

Then

$$
\left|a_{-t+v p}^{(-t / p)}\right| \leqq\left(\begin{array}{c}
2 t / p \\
v
\end{array}\right)
$$

$$
\begin{aligned}
n\left|a_{-t+n p}^{(-t / p)}\right| & \leqq 2\left[\frac{t}{p}+\left(\frac{t}{p}-1\right)\left|a_{-t+p}^{(-t / p)}\right|+\cdots+\left(\frac{t}{p}-(n-1)\right)\left|a_{-t+(n-1)}^{(-t p)}\right|\right] \\
& \leqq 2\left[\frac{t}{p}+\left(\frac{t}{p}-1\right)\left(\begin{array}{c}
2 t / p \\
1
\end{array}\right)+\cdots+\left(\frac{t}{p}-(n-1)\right)\left(\begin{array}{c}
2 t / p \\
n-1
\end{array}\right)\right] \\
& =n\left(\begin{array}{c}
2 t / p \\
n
\end{array}\right)
\end{aligned}
$$

and therefore

$$
\left|a_{-t+n p}^{(-t / p)}\right| \leqq\left(\begin{array}{c}
2 t / p \\
n
\end{array}\right)
$$

If $n-1=t / p$, again making the inductive assumption, we have

so

$$
\begin{aligned}
n\left|a_{-t+n p}^{(-t / p)}\right| & \leqq 2\left[\frac{t}{p}\left(\begin{array}{c}
\frac{t}{p}-1
\end{array}\right)\left(\begin{array}{c}
2 t / p \\
1
\end{array}\right)+\cdots+\left(\begin{array}{c}
2 t / p \\
n-2
\end{array}\right)\right] \\
& =(n-1)\left(\begin{array}{l}
2 t / p \\
n-1
\end{array}\right)
\end{aligned}
$$

$$
\begin{aligned}
\left|a_{-t+n p}^{(-t / p)}\right| & \leqq \frac{(n-1)}{n}\left(\begin{array}{c}
2 t / p \\
n-1
\end{array}\right) \\
& =\frac{(n-1)}{n}\left(\begin{array}{c}
2(n-1) \\
n-1
\end{array}\right) \\
& =\frac{(n-1)}{n} \frac{[2(n-1)] !}{(n-1) !(n-1) !}=\left(\begin{array}{c}
2 t / p \\
n
\end{array}\right) .
\end{aligned}
$$


These inequalities are sharp as is shown by

$$
\left(\frac{z}{\left(1+z^{p}\right)^{2 / p}}\right)^{-t}=\frac{1}{z^{t}}\left[1+\sum_{v=1}^{\infty}\left(\begin{array}{c}
2 t / p \\
v
\end{array}\right) z^{v p}\right] .
$$

RemarK 4.1. Every function $f_{p}$ in $S_{p}$ may be expressed as $f_{p}(z)=f\left(z^{p}\right)^{1 / p}, f \in S$, and every function $\tilde{f}_{p} \in \Sigma_{p}$ may be expressed as $f_{p}(z)=f_{p}(1 / z)^{-1}, f_{p} \in S_{p}$. Also if $f \in S$, then $f\left(z^{p}\right)^{1 / p} \in S_{p}$, and if $f_{p} \in S_{p}$, then $f_{p}(1 / z)^{-1} \in \Sigma_{p}$. Hence (4) and (6) of $\S$ II hold for $S_{p}$ and $\Sigma_{p}^{-1}$. Thus, as in $\S$ III, we have the following theorems.

COROLlary 4.1. If $f_{p} \in S_{p}$ and $t>0$, then we have the sharp inequality

$$
\left|a_{-t+n p}^{(-t)}\right| \leqq\left(\begin{array}{c}
2 t / p \\
n
\end{array}\right)
$$

for all $n$ such that $p(n-1)-t \leqq 0$.

Proof. This restatement of Theorem 4.1 follows from Remark 4.1.

THEOREM 4.2. If $\phi_{p} \in S_{p}^{-1}, \phi_{p}(w)=\sum_{v=0}^{\infty} b_{v p+1} w^{v p+1}$, then we have the sharp inequality

$$
\left|b_{n p+1}\right| \leqq \frac{1}{n p+1}\left(\begin{array}{c}
2(n p+1) / p \\
n
\end{array}\right), \quad n=1,2, \cdots
$$

Proof. See the proof of Theorem 3.8.

Corollary 4.2 (CF. TheORem 3.3). If $\phi \in S^{-1}$, then we have the sharp inequality

$$
\left|b_{n}\right| \leqq \frac{1}{n}\left(\begin{array}{c}
2 n \\
n-1
\end{array}\right), \quad n=1,2, \cdots .
$$

THEOREM 4.3. If $\tilde{\phi}_{p} \in \Sigma_{p}^{-1}, p \geqq 1, \tilde{\phi}_{p}(w)=w+\sum_{n=1}^{\infty} \tilde{b}_{-1+n p} w^{-1+n p}$, then we have the sharp inequality

$$
\left|\tilde{b}_{-1+n p}\right| \leqq \frac{1}{n p-1}\left(\begin{array}{c}
2(n p-1) / p \\
n
\end{array}\right), \quad n=1,2, \cdots .
$$

Proof. See the proof of Theorem 3.10.

THEOREM 4.4 (C.F THEOREM 3.4). If $\tilde{\phi} \in \Sigma^{-1}$, then

$$
\begin{aligned}
& \left|\tilde{b}_{n}\right| \leqq \frac{1}{n}\left(\begin{array}{c}
n \\
n+1
\end{array}\right), \quad n=1,2, \cdots, \\
& \left|\tilde{b}_{0}\right| \leqq 2,
\end{aligned}
$$

and these inequalities are sharp. 
Proof. The first inequality follows from Theorem 4.3. To see that $\left|\tilde{b}_{0}\right| \leqq 2$ consider (3):

$$
\begin{aligned}
\sum_{t=-\infty}^{\infty} b_{0}^{(t)} z^{-t-1} & =\frac{f^{\prime}(z)}{f(z)} \\
& =\frac{1}{z}+a_{2}+\cdots
\end{aligned}
$$

By (6)

$$
\begin{aligned}
\left|\tilde{b}_{0}\right| & \leqq \max _{\phi \in S^{-1}}\left|b_{0}^{(-1)}\right| \\
& =\max _{f \in S}\left|a_{2}\right| \\
& =2 .
\end{aligned}
$$

Finally, the Theorem of Prawitz (Theorem 3.6) is valid for $S$ and thus Corollaries 3.1 and 3.2 are also valid for $f \in S$.

V. Concluding remarks. Consider the set of functions

$$
S^{* \prime}=\left\{f: f \in S^{*} ;\left|a_{-v}^{(-t)}\right|=\left|a_{v}^{(-t)}\right|, v=1, \cdots, t-1\right\} .
$$

If $f \in S^{* \prime}$, then, for $p=1$, equation (16) becomes, for $n>2 t$,

$$
\begin{aligned}
n^{2}\left|a_{-t+n}^{(-t)}\right| & \leqq 4 t^{2}+4 t \sum_{v=1}^{n-1}(t-v)\left|a_{-t+v}^{(-t)}\right|^{2} \\
& \leqq 4 t^{2} .
\end{aligned}
$$

Therefore

$$
\left|a_{n}^{(-t)}\right| \leqq \frac{2 t}{n+t}, \quad n=t+1, \cdots
$$

This inequality is sharp:

$$
\left(\frac{z}{\left(1+z^{n+1}\right)^{2 /(n+t)}}\right)^{-t}=\frac{1}{z^{t}}+\frac{2 t}{n+t} z^{n}+\cdots .
$$

This, along with equation (13) and the fact that $\left(z /(1+z)^{2}\right)^{-t}$ is an extremal function, leads us to conjecture that if $f \in S^{*}$ then for $t>0$

$$
\left|a_{n}^{(-t)}\right| \leqq\left(\begin{array}{c}
2 t \\
t+n
\end{array}\right), \quad n=1, \cdots, t
$$

and

$$
\left|a_{n}^{(-t)}\right| \leqq \frac{2 t}{t+n}, \quad n=t+1, \cdots
$$


Equation (13) and (24) and the extremal function $\left(z /(1+z)^{2}\right)^{-t}$ lead us to conjecture that if $f \in S$ then for $t>0$

$$
\left|a_{n}^{(-t)}\right| \leqq\left(\begin{array}{c}
2 t \\
t+n
\end{array}\right), \quad n=1, \cdots, t,
$$

but Garabedian and Schiffer [5] have shown that if $\tilde{f} \in \Sigma$ then $\left|\tilde{a}_{3}\right| \leqq 1 / 2+e^{-6}$ is sharp and so we cannot make any general conjecture about the bounds for

$$
\left|a_{n}^{(-t)}\right|, \quad n=t+1, \cdots
$$

\section{REFERENCES}

1. L. Bieberbach, Über die Koeffizienten derjenigen Potenzreihan, welche eine schlicht Abbildung des Einheitskreises vermittein, S.-B. Preuss. Akad. Wiss. Berlin (1916), 940-955.

2. Z. Charzynski and M. Schiffer, A new proof of the Bieberbach conjecture for the fourth coefficient, Arch. Rational Mech. Anal. 5 (1960), 187-193.

3. - A geometric proof of the Bieberbach conjecture for the fourth coefficient, Scripta Math. 25 (1960), 173-181.

4. J. Clunie, On meromorphic schlicht functions, J. London Math. Soc. 34 (1959), 215-216.

5. P. R. Garabedian and M. Schiffer, A coefficient inequality for schlicht functions, Ann. of Math. 61 (1955), 116-136.

6. - A proof of the Bieberbach conjecture for the fourth coefficient, Arch. Rational Mech. Anal. 4 (1955), 427-465.

7. G. M. Golusin, Geometrische Funktionentheorie, Deutscher Verlag der Wissenschaften, Berlin, 1957.

8. Einar Hille, Analytic function theory, Vol. II, Ginn, New York, 1962, 346-359.

9. Eri Jabotinsky, Representation of functions by matrices. Application to Faber polynomials, Proc. Amer. Math. Soc. 4 (1953), 546-553.

10. Karl Lowner, Untersuchengen über schlicht konforme Abbildungen des Einheitskreises. I Math. Ann. 89 (1923), 103-121.

11. Z. Nehari and E. Netanyahu, Coefficients of meromorphic schlicht functions, Proc. Amer. Math. Soc. 8 (1957), 15-23.

12. E. Netanyahu, Extremal problems for Schlicht functions in the exterior of the unit circle, Canad. J. Math. 17 (1965), 335-341.

13. R. Nevanlinna, Uber die konforme Abbildung von Sterngebieten, Finska Vetenskaps Soc. Forhankl (A) 63 (1921-1922), No. 7.

14. H. Prawitz, Uber Mittelwerte analytischer Funktionen, Ark. Mat. Astronom. Fys. 20 (1927-1928), 1-12.

15. W. C. Royster, Extremal problems for functions starlike in the exterior of the unit circle, Canad. J. Math. 14 (1962), 540-551.

16. - Meromorphic starlike multivalent functions, Trans. Amer. Math. Soc. 107 (1963), 300-308.

17. A. C. Schaeffer and D. C. Spencer, The coefficients of schlicht functions. I, Duke Math. J. 10 (1943), 611-635.

18. —, The coefficients of schlicht functions. II, Duke Math. J. 12 (1945), 107-125.

19. —, A variational method in conformal mapping, Duke Math. J. 14 (1947), 949-966.

20. — Coefficient regions for schlicht functions, Amer. Math. Soc. Colloq. Publ. Vol. 35, Amer. Math. Soc., Providence, R. I., 1950. 
21. A. C. Schaeffer, M. Schiffer and D. C. Spencer, The coefficient regions of schlicht functions, Duke Math. J. 16 (1949), 493-527.

22. M. Schiffer, $A$ method of variation within the family of simple functions, Proc. London Math. Soc. 44 (1938), 432-449.

23. $450-452$.

24. - Variation of the Green's function and theory of the p-valued functions, Amer. J. Math. 65 (1943), 341-360.

25. - Application of variational methods in the theory of conformal mappings, Proc. Sympos. Appl. Math. Vol. 8, pp. 93-113, Amer. Math. Soc., Providence, R. I., 1958.

26. - Extremum problems and variational methods in conformal mapping, Proc. Internat. Congr. Math. 1958, Cambridge Univ. Press, New York, 1960.

27. G. Springer, The coefficient problem for schlicht mappings of the exterior of the unit circle, Trans. Amer. Math. Soc. 70 (1951), 421-450.

Florida State University,

TAllahasse, Florida 